\title{
La tensión estandarización-diferenciación en las políticas culturales. El caso de España y Francia
}

\author{
Lluís Bonet \\ Universidad de Barcelona \\ Ibonet@ub.edu
}

Emmanuel Négrier

CNRS. Université de Montpellier 1

negrier@univ-montp1.fr

\begin{abstract}
Resumen
A partir de una interpretación "intergubernamental" de las políticas públicas, el presente artículo estudia las tendencias hacia la diferenciación y la estandarización, tomando como caso comparado de estudio la política cultural franco-española, dos modelos con similitudes y diferencias especialmente relevantes. Para ello, se parte de un esquema analítico que combina cinco ejes: la configuración institucional, los instrumentos de in tervención, la distribución por niveles de gobierno, las lógicas con capacidad de influencia y las prioridades, objetivos y valores de las políticas. Una primera hipótesis es que la doble tendencia hacia la diferenciación o hacia la estandarización no se limita al diálogo/tensión entre los Estados y los organismos supranacionales, sino que integran al conjunto de interacciones con los niveles subestatales; y ello más allá de la estructura federal o unitaria del Estado. La segunda hipótesis es que en cada nivel de gobierno se dan tendencias hacia la diferenciación y hacia la estandarización. El análisis de los argumentos de legitimación y eficiencia en un conjunto de observaciones contextualizadas permite contrastar las hipótesis.
\end{abstract}

Palabras clave

Políticas culturales, legitimidad, eficiencia, gobierno multinivel, investigación comparada.

\section{The tension between standardization and differentiation in cultural policy. The case of Spain and France}

\begin{abstract}
From an "intergovernmental" interpretation of public policies, this paper examines the trends towards differentiation and standardization. It uses a comparative case study, the cultural policies of France and Spain, with quite relevant models of similarities and differences. To do it, develops an analytical framework that combines five axis of governmental analysis: the institutional settings, the instruments of intervention, the distribution by levels of government, the lobby capacity of actors and logics, and the priorities, objectives and values of cultural policies. One hypothesis is that the twin trends towards differentiation or toward standardization is not limited to dialogue / tension between states and supranational bodies, but up to all interactions with subnational levels, and therefore beyond the federal or unitary state. The second hypothesis is that at every level of government there are tendencies toward differentiation and standardization. The analysis of the arguments of legitimacy and efficiency to a set of observations enables contextualized hypotheses.
\end{abstract}




\section{INTRODUCCIÓN}

Uno de los fenómenos más frecuentes en la implementación de los modelos de gestión de las políticas públicas contemporáneas en Europa es su creciente convergencia (Wallace y Wallace, 2000; Kassim et al., 2001). Diversas razones explican este comportamiento, desde la asunción del acervo comunitario y su posterior desarrollo por parte del entramado institucional y normativo de la Unión (Radaelli, 2003; Saurruger y Surel, 2006) hasta la adaptación a los retos de la globalización (Dezalay y Garth, 2002; Drezner, 2005). En las diversas políticas públicas, los actores tienden a reorganizarse o a incorporar formas de intervención a escala europea, sin que esto implique la desaparición de los valores y culturas organizativas nacionales (Fligstein, 2008). Por un lado, se da un cierto isomorfismo mimético, una globalización instrumental que presiona a los actores en busca de modelos o recetas "que funcionan" en otros países (DiMaggio y Powell, 1991), pues, en la medida que la globalización nos hace más interdependientes y conscientes de compartir los mismos retos, incide asimismo en homogeneizar la respuesta a dichos problemas. Por otro lado, la interpenetración de los instrumentos y las estrategias políticas es coherente con la creciente importancia de las normas europeas e internacionales (Knill y Lehmkuhl, 2002).

Sin embargo, una interpretación bastante común relaciona la inestabilidad del sistema actual con la tensión existente entre niveles de intervención gubernamental (Knill, 1998). Por una parte, los Estados intentan encontrar estrategias específicas, adaptadas a la propia singularidad, para luchar contra los problemas generados por la globalización, en una tentativa para posicionarse por delante de los demás (Lenschow et al., 2005). Por otra, las instituciones supranacionales pretenden influir en los sistemas nacionales a través de estrategias de normalización y estandarización, para lograr efectos de escala que resuelvan los problemas comunes (Evans, 2004). Los factores nacionales que homogeneízan las distintas políticas públicas y sus modelos de gestión son evidentes, pero de forma creciente se relativizan por fenómenos de diferenciación interna hacia abajo (consecuencia de la experiencia y la relación entre niveles de poder) y por la hibridación externa fruto de los acuerdos e influencias a nivel comunitario o internacional (Radaelli y Schmidt, 2004).

El objeto del presente trabajo consiste en analizar la interpretación "intergubernamental” de las políticas públicas aplicada a una política sectorial específica: la cultural. La implementación de una política cultural democrática explícita toma fuerza en Europa y en algunos otros países occidentales después de la Segunda Guerra Mundial. La intervención del Estado en el ámbito artístico y del patrimonio cultural se legitima en fines como: la democratización del acceso a la cultura, el apoyo a la creación, el desarrollo de una industria cultural nacional, la formación de sus profesionales, o el fortalecimiento de la identidad local y nacional, entre otros (Cummings y Katz, 1987). La extensión de esta nueva categoría de intervención gubernamental ha dado lugar al nacimiento y consolidación de estrategias, departamentos y servicios específicos, así como de profesionales especializados en las distintas administraciones naciona-

54 les, regionales y locales. Las grandes orientaciones políticas, las áreas de intervención 
y en cierta medida también las herramientas específicas de las políticas culturales siguen parámetros bastante homogéneos a escala internacional bajo el impulso de instituciones intergubernamentales como la Organización de las Naciones Unidas para la Educación la Ciencia o la Cultura (UNESCO) o el Consejo de Europa. En cambio, los modelos e instrumentos específicos de intervención, así como el valor de los recursos financieros puestos a disposición, muestran divergencias mayores en función de la orientación ideológica dominante, la fortaleza o percepción de debilidad de la identidad cultural nacional, la capacidad de influencia corporativa de los sectores económicos o profesionales, o la tradición y modelos de intervención pública dominantes en cada país (Audet y Saint-Pierre, 2010).

A partir de una interpretación "intergubernamental” de las políticas públicas, el presente artículo toma como caso comparado de estudio la política cultural francoespañola, dos modelos con similitudes y diferencias especialmente relevantes. Nuestra primera hipótesis es que la doble tendencia hacia la diferenciación o hacia la estandarización no se limita al diálogo/tensión entre los Estados y los organismos supranacionales, sino que integra asimismo al conjunto de interacciones con los niveles subestatales; y ello más allá de la estructura federal o unitaria del Estado. Nuestra segunda hipótesis es que en cada nivel de gobierno se dan tendencias hacia la diferenciación y hacia la estandarización, entremezcladas con la tensión entre legitimidad y eficiencia. Para contrastar dichas hipótesis, vamos a utilizar un conjunto de observaciones contextualizadas comparadas sobre políticas públicas de cultura de España y Francia procedentes de diversos estudios y estadísticas recientes (Ariño et al., 2005; Bonet y Négrier, 2008, 2011; Delvainquière, 2010; Dubois, 2010; Ministère de la Culture et de la Communication, 2010; Ministerio de Cultura, 2010; Rubio Aróstegui, 2003, 2005, 2008; Villarroya, 2010).

La propuesta parte de la premisa que la tensión entre la estandarización y la diferenciación de las políticas públicas no se puede resolver simplemente con una respuesta de tipo instrumental, como exigiría una visión estrecha de la nueva gestión pública (Dunn y Miller, 2007), pues si la respuesta del instrumento es inadecuada es porque refleja tanto los desafíos de racionalización de la acción pública, como las cuestiones relativas a su legitimidad. En otras palabras, se trata de una constante interacción de doble voltaje, razón por la cual nuestra comparación de dichas dimensiones refleja, de forma más o menos explícita, la cuestión de la legitimidad y de la eficiencia (Muller, 2009). Para profundizar en ello, se propone un esquema analítico que combina cinco ejes: la configuración institucional, los instrumentos de intervención, la distribución de competencias por niveles de gobierno, las lógicas con capacidad de influencia y las prioridades, objetivos y valores de las políticas. Mientras los tres primeros, más institucionales, permiten una comparación empírica de nuestras hipótesis, los dos últimos admiten solo una primera evaluación de la doble interacción entre diferenciación y estandarización, y entre legitimidad y eficiencia.

Cabe tener en cuenta que el ámbito de las políticas culturales presenta una singularidad clara. Por un lado, sigue las mismas tendencias homogeneizadoras de las 
demás políticas sociales; pero, por otro, es un extraordinario exponente de la necesidad de diferenciación. Algunas razones ayudan a explicarlo.

El campo de intervención cultural y los paradigmas que dirigen sus políticas, así como los beneficiarios de las mismas, son mucho más etéreos (basados en las externalidades) y de impacto directo más reducido o a largo plazo que el de otras políticas sociales, como las de educación, sanidad o servicios sociales, que tienen un carácter mucho más universal (bienes públicos). Esta es la razón por la que las políticas culturales tienen una mayor necesidad de legitimación; situación especialmente patente en momentos de recorte presupuestario. De todas formas, la percepción social sobre su impacto oscila entre la consideración de lujo irrelevante (guinda del pastel) y de columna vertebral de desarrollo -en el espíritu que Amartya Sen define el desarrollo de las capacidades (Sen, 1989)-. En todo caso, el escaso desarrollo de modelos contrastados de evaluación hace que a menudo los responsables culturales deban responder a acusaciones de clientelismo, corporativismo, arbitrariedad, subjetividad en los criterios de decisión o falta de indicadores de impacto simbólico. Este último aspecto es clave, pues en muchos casos - desde el Guggenheim a los grandes equipamientos que cada presidente francés lega a París- los beneficios sociales obtenidos se asientan en la singularidad del proceso, hecho que hace muy difícil prever a priori los impactos de la acción. De esta manera, el debate entre eficiencia y legitimación está permanentemente servido, pues avanza desde las grandes capitales o los proyectos más singulares a poblaciones y equipamientos totalmente periféricos (¿Cómo se explica si no la construcción de centros culturales sobredimensionados en poblaciones muy pequeñas?).

Otro aspecto bastante específico es su enraizada dimensión territorial, pues la cultura es una de las herramientas de identidad clave utilizada por instituciones y políticos locales para fortalecerse frente a la globalización y a otras tendencias homogeneizadoras (Bonet y Négrier, 2010). En muchos países occidentales, no solo en España y Francia, ésta es una competencia concurrente, donde el nivel de administración que más gasta es el menos obligado por ley a dedicar recursos a la misma.? ? De todas formas, la dialéctica entre armonización y diferenciación atañe a todos los niveles de gobierno. Por otro lado, la defensa de la autonomía de la cultura frente al poder público es una constante que atraviesa históricamente el desarrollo de las políticas culturales (Vestheim, 2009).

En otros aspectos, la política cultural presenta grandes semejanzas con otras políticas sociales (Gomà y Subirats, 1998). Por ejemplo, buena parte de los programas responden a lógicas profesionales específicas, construidas a lo largo de décadas, que limitan la capacidad de cada nuevo gobierno para transformar los programas específicos. Bibliotecarios, conservadores de museos, archiveros o técnicos de cultura disponen de un amplia autonomía profesional, fortalecida por una deontología explícita y el bagaje académico; autonomía que solo la extrema derecha ha osado cuestionar. Por ejemplo, al ganar la extrema derecha francesa las municipales en Orange se pro56 dujo un conflicto sobre una cuestión aparentemente técnica -la selección del mate- 
rial de las bibliotecas públicas- que rompía con la autonomía profesional (BressatBodet, 1999). Los límites entre el espacio ideológico y el espacio definido por la academia y la profesión, así como los criterios construidos (en alguna ocasión justificados fundamentalmente por intereses gremiales) evolucionan al ritmo -casi siempre muy lento- al que se transforma el sistema de valores dominantes.

\section{MODELOS DE POLÍTICA CULTURAL Y SU ADAPTACIÓN AL CASO FRANCÉS Y ESPAÑOL}

España y Francia son dos excelentes casos de estudio para evaluar el desarrollo de sus respectivos sistemas de gobernanza y, en especial, las tendencias de diferenciación y estandarización de sus políticas culturales. La convergencia inicial de sus modelos de administración y, al mismo tiempo, las diferencias en su desarrollo histórico -y muy en particular en la relación centro-periferia- son evidentes.

Aun aceptando la actual gran hibridación entre modelos y la dificultad del análisis comparado a escala internacional (Belfiore, 2004), la comunidad académica utiliza en la mayor parte de ejercicios de comparación internacional las cuatro grandes tipologías institucionales clásicas de política cultural (Chartrand y McCaughey, 1989). Dicha clasificación, centrada en los aspectos institucionales y en los mecanismos de financiación gubernamental, es bastante simple, didáctica y popular en el ámbito académico. En este esquema, Francia y España forman parte del modelo "arquitecto" al contar con departamentos de cultura integrados plenamente en la estructura de la administración (ministerios o direcciones de cultura), con mecanismos de intervención directa legitimados democráticamente (Bouzada, 2007; Dubois, 2010).

\section{CUADRO 1}

Modelos institucionales de política cultural según Chartrand y McCaughey (1989)

\begin{tabular}{|c|c|c|c|}
\hline Modelo & País ejemplo & Institución clave & Sistema \\
\hline Facilitador & EE.UU. & Individuos, NEA-NEH & Beneficio fiscal \\
\hline Patrocinador & Reino Unido & Consejo de las artes & $\begin{array}{c}\text { Gestión a distancia } \\
\text { Arm's length }\end{array}$ \\
\hline Arquitecto & Francia & Ministerio & Intervención directa \\
\hline Ingeniero & URSS & Ministerio, Sindicatos & Imposición \\
\hline
\end{tabular}

Fuente: Chartrand, H. y McCaughey, C. (1989).

Sin embargo, un ejercicio más exhaustivo de análisis comparado de las políticas públicas debería ir más allá de la configuración institucional o de los mecanismos de financiación gubernamental, para profundizar en aspectos como la estructura del Estado, los modelos de gestión, los valores de la acción pública o las estrategias e instrumentos utilizados. Los países no se clasifican en razón de su prefiguración respecto de un modelo político-institucional de referencia, sino en la combinación de 
muchas variables más. Para facilitar una mirada más compleja y evaluar la evolución de las diferencias y similitudes entre los países en el ámbito de las políticas culturales, se propone un nuevo esquema analítico conformado alrededor de cinco grandes ejes:

A) Configuración institucional de la intervención gubernamental (principal o secundaria, dada la creciente hibridación entre modelos).

- Intervención directa (Departamentos de cultura).

- Gestión a distancia (arm's length) de las instituciones y regímenes de subvenciones.

- Beneficios fiscales (orientados a la oferta o a la demanda).

B) Importancia relativa de los distintos instrumentos de intervención utilizados

- Regulación (protección del patrimonio, propiedad intelectual, cuotas para productos nacionales, regulación del mercado y los precios, etc.).

- Provisión gubernamental de bienes y servicios (titularidad y/o gestión directa de museos, bibliotecas, teatros, festivales u otros proyectos).

- Sistema de incentivos directos e indirectos (subvenciones, ventajas fiscales, adquisiciones o capital riesgo, entre otros).

- Información y estrategias relacionales o de mediación (asesoramiento, reconocimientos públicos, ventanilla única, marcas de calidad, etc.).

- Inspección y sanción (conflicto respecto a los valores y prácticas ciudadanas, proceso de adaptación, capacidad real de implementación).

C) Distribución de responsabilidades y competencias entre niveles de gobierno.

- Capacidad legislativa.

- Distribución de los recursos presupuestarios y de carácter obligatorio/voluntario.

- Liderazgo conceptual (la capacidad de imponer nuevas prioridades y paradigmas).

D) Las lógicas con capacidad de influencia (asimétrica relación de fuerzas de los distintos agentes involucrados). 
- Sistema institucional y cultura política (normas, burocracia...).

- Las fuerzas del mercado.

- Sector no lucrativo.

- La política (ideología y partidos políticas).

E) Prioridades, objetivos y valores.

- Metas intrínsecas frente a metas extrínsecas.

- Entre los sectores (patrimonio, artes creativas, industrias culturales...) y estrategias (educación artística, formación profesional, creación, producción, difusión y consumo, conservación).

La combinación a geometría variable de este conjunto de variables permite desgajarse de la aparente homogeneidad del modelo institucional de partida -en este caso, el modelo "arquitecto", común para España y Francia-, para analizar las tendencias de diferenciación o estandarización existentes en las formas de implementación de las políticas públicas. Para ello, es necesaria una sintética valoración de las características de los dos países escogidos respecto a cada uno de los cinco ejes de referencia. Pero mientras que los tres primeros, más institucionales, permiten una comparación empírica de nuestras hipótesis, los dos últimos se tratarán al estudiar la tensión en la construcción dialéctica del discurso entre legitimidad y eficiencia en los procesos de estandarización o diferenciación de las políticas culturales.

\section{A) Configuración institucional de la intervención gubernamental}

El desarrollo y evolución de las políticas culturales españolas no puede sustraerse de la particular cultura política construida en España desde la instauración del Estado autonómico a partir de la Constitución de 1978 (Prieto de Pedro, 1995; Moreno, 2001). El sistema institucional de intervención gubernamental en la mayoría de países europeos se basa en la intervención directa del ejecutivo que consigue la confianza parlamentaria (aunque en Francia el Presidente de la República tiene un poder de contrapeso). La política cultural es dirigida por organismos administrativos liderados por políticos en representación de la mayoría gubernamental, asesorados por funcionarios y personal técnico a su cargo. La existencia en España de un único organismo de gestión a distancia, según el principio del arm's length de tradición anglosajona, como es el Consejo Nacional de la Cultura y las Artes de Cataluña (CONCA), representa una excepción en el entramado cultural institucional.

Por su lado, la política cultural francesa se caracteriza por su centralismo y, como España, por su elevado voluntarismo institucional. Sin embargo, una mirada más 
atenta a las estrategias e instrumentos implementados en las últimas décadas permite matizar el modelo. Existen distintas formas de intervención, instrumentos directos e indirectos, que no siempre responden a una lógica jerárquica. El auge de los poderes locales diluye en parte la tradicional mentalidad orgánica y centralista.

En ambos casos, la intervención gubernamental se plasma en la intervención directa y se ejemplariza en el volumen de gasto público. Esto explica la menor relevancia de los beneficios fiscales en comparación con Estados Unidos o el Reino Unido (Cummings y Katz, 1987). Por ejemplo, en España, los beneficios fiscales solo representan el $18 \%$ sobre el total de la aportación gubernamental a la cultura del año 2008, de los cuales dos terceras partes corresponden a deducciones por IVA (las publicaciones y en menor medida los espectáculos gozan de deducciones significativas), las exenciones sobre el impuesto de sociedades representan un 30\%, y solo un $3 \%$ procede del IRPF. La situación francesa es parecida en lo que concierne a las deducciones del IVA (publicaciones y espectáculos están gravados con el 5,5\%, respecto a un tipo general del 19,5\%), pero no así respecto a los beneficios del impuesto de sociedades. Éstos son bastante más generosos desde la aplicación de la Ley de mecenazgo de 2003 al permitir una deducción general del 60\% de la donación, proporción que alcanza el 90\% si se trata de la adquisición de bienes de interés nacional.

Desde finales de los años ochenta, cuando Chartrand y McCaughey definen el modelo "arquitecto", España y Francia han experimentado nuevas formas de configurar su política cultural. La matriz básica (el modelo de intervención directa) no se ha transformado significativamente, pero la implementación de generosos beneficios fiscales en Francia o la creación del CONCA en España muestran una diversificación de los actores con capacidad de decisión. En el modelo resultante, cada vez más híbrido, el actor gubernamental dominante cede ciertas áreas de responsabilidad a las empresas o a la sociedad civil, o a un organismo con mucho menos control político (caso del Consejo de las Artes). En cierta medida, la legitimidad del Estado-nación continental, basado en la representatividad democrática del gobierno, se traslada lentamente hacia actores externos que aportan nueva legitimidad y eficiencia en la consecución de los fines a alcanzar.

\section{B) Importancia relativa de los distintos instrumentos utilizados}

La regulación es una competencia exclusiva de los poderes públicos, aunque en algunos países se fomente la reglamentación o los códigos de conducta profesionales o sectoriales internos (por ejemplo, en la fijación del precio del libro u otros). En la tradición continental más reglamentista, compartida por España y Francia, es importante distinguir entre el marco normativo general formulado por los distintos entes con capacidad legislativa (a nivel europeo, estatal o subestatal) en los ámbitos fiscal, laboral, económico o social, y la legislación más específica que regula el sector cultural. Estos últimos pueden desarrollar los marcos anteriores (como la concreción de 60 determinados beneficios fiscales o laborales) o comprender ámbitos particulares 
como, entre otros, la propiedad intelectual y los derechos de autor, la protección del patrimonio y su defensa frente a la expoliación, la legislación de espectáculos, la regulación de marcas de calidad, la definición de cuotas para las obras europeas en la difusión televisiva o radiofónica, o el precio único del libro. A pesar de los matices propios de las distintas culturas jurídicas, la comparación entre sistemas nacionales conduce hacia una creciente convergencia, impuesta en buena parte por directivas europeas (Dubois y Négrier, 1999; Gray, 1996; Benhamou, 2006). Sin embargo, subsisten singularidades nacionales como el estatuto francés para los intermitentes del espectáculo, un sistema sufragado por los fondos generales de la seguridad social que se hace cargo de las prestaciones de paro durante los largos periodos en que un profesional del espectáculo no está activo. Dicho régimen, mucho más generoso, difiere claramente de la cobertura de los trabajadores discontinuos y de temporada del régimen español.

El otro gran ámbito de preeminencia gubernamental corresponde a la provisión directa. Ésta, tanto en el caso español como en el francés, concentra los recursos presupuestarios disponibles. En cambio, el peso de los incentivos, y en especial de las estrategias de mediación o información, es bastante menos importante en los países latinos de Europa que en el mundo anglosajón (Cummings y Katz, 1987; Belfiore, 2004).

El análisis de la naturaleza económica del gasto permite distinguir entre las partidas referidas a la provisión directa (personal, gastos en bienes y servicios, e inversión real en equipamiento propio) de las transferencias corrientes o de capital a otros organismos. En España, el primer grupo de partidas representa el 70,1\% del gasto cultural total efectuado por el conjunto de entes gubernamentales (año 2008). Pero mientras que en la administración central y en las administraciones locales dicha proporción alcanza el 71,3\% y el 77,4\%, respectivamente, en el caso de las Comunidades Autónomas es algo menor pues "solo" representa el 58,3\% (Ministerio de Cultura, 2010). Si los datos agregados permitieran distinguir entre las transferencias a organismos autónomos dependientes de la propia administración de aquellas transferencias a los creadores o a otras entidades públicas o privadas, las proporciones serían aun más altas. Así pues, más allá de una retórica que dice querer fortalecer el tejido cultural, la administración española es altamente autorreferencial, mucho más orientada a prestar servicios directos a través de sus equipamientos o proyectos que con una voluntad real de incentivar la actividad de otras instituciones o actores. En Francia, la situación es similar: el Ministerio de Cultura y Comunicación solo destina el 29\% de su presupuesto total a subvenciones y ayudas, mientras que los costes operativos (43\%) y los gastos en personal (22\%) se llevan la mayor parte de los recursos disponibles. Las ciudades de más de 10.000 habitantes dedican solo el $19 \%$ a transferencias y las entidades de cooperación inter-municipal el 23\% (Ministère de la Culture et de la Communication, 2010).

Como complemento del revelador análisis presupuestario, merece la pena distinguir a nivel funcional las diversas formas de control administrativo sobre los entes 
descentralizados, de responsabilidad compartida con otras administraciones, y el resto de instituciones, proyectos o personas merecedores de apoyo y/o subvención. Las formas de tutela política y de control sobre la gestión, más allá de la forma jurídica de cada organismo específico, son claramente distintas en cada uno de los siguientes casos: entes descentralizados de la propia administración (empresas públicas, organismos autónomos administrativos o comerciales, etc.), servicios de titularidad pública externalizados a empresas o entidades, organismos bajo tutela compartida gestionados a través de consorcio u otra forma de representación directa en los órganos de decisión, o subvención a otros niveles de gobierno, instituciones culturales de base o a creadores individuales. En estos últimos casos, los diversos mecanismos de subvención utilizados (automática, condicionada, competitiva) describen la filosofía de la relación entre el ente gubernamental y los proyectos culturales.

Así pues, existe una gran modulación de formas de control y ascendencia por parte del estado, de menor a mayor intensidad, y con compromisos anualizados o a largo plazo. A pesar del tradicional, y cada vez más exigente, control económico y administrativo por parte de la intervención pública, la progresiva penetración de las reglas de la nueva gestión pública conduce hacia una mayor preocupación por la eficiencia (Hood, 2000). Esto se hace patente en los contratos-programas con las instituciones culturales, de larga tradición en Francia y más recientes en España, con la explicitación de indicadores de evaluación. En Francia, el modelo de evaluación por indicadores a instituciones públicas representa el 20\% del total del presupuesto cultural, afectando desde al Centro Nacional del Cine o la Comedia Francesa, a los castillos del Loire. En España de momento solo alcanza a algunos grandes equipamientos emblemáticos (Gran Teatre del Liceu, Museo del Prado, Mercat de les Flors, etc.). A esta nueva tendencia se debe unir la creciente selección de los nuevos directores de los grandes museos, teatros, auditorios y compañías estables a partir de concursos abiertos (Reina Sofía, Ballet Nacional de España, Auditori de Barcelona, etc.) con la presentación de un plan programático e indicadores objetivos de selección. La incorporación de estos modelos está revolucionando la vieja tradición hispánica de selección a dedo y evaluación por criterios de fidelidad política en lugar de mérito, capacidad o eficiencia. De momento, ambos modelos conviven, con crisis periódicas cada vez que el viejo sistema muestra su cara oscura (como las destituciones de los directores del MuVIM de Valencia, el Daz de Salamanca, o hace algunos años del CGAC de Santiago), pues más allá de la retórica eficientista y de respecto a la autonomía de los profesionales, los valores de fidelidad, de no salirse de la norma o despuntar y de opacidad están aún muy enraizados en la cultura política española.

En referencia a la subvención, ambos países mantienen la discrecionalidad de los responsables políticos basada en las recomendaciones de comisiones consultivas de evaluación. Este modelo, sin embargo, empieza a entrar en crisis gracias al influjo de los sistemas de evaluación europeos. Los programas Media o Cultura, entre otros, están imponiendo mayor transparencia en los criterios de evaluación y de anonimato en los evaluadores que trastocan el paradigma mucho más clientelar que caracteriza 62 tradicionalmente la relación entre subvencionador y subvencionado. Un caso emble- 
mático, con amplia trascendencia en la formas de prepararse para competir, es el proceso de selección de las ciudades capitales europeas de la cultura. Fue así en el caso de Francia para designar la capital para el año 2013, y lo es en España para el año 2016. La confrontación que subyace no solamente transforma el proceso de selección, sino también la forma de diseñar los programas culturales con la incorporación de objetivos e indicadores de evaluación precisos.

Otro aspecto que describe la filosofía de la relación entre el ente gubernamental y el receptor de la ayuda es el predominio de las subvenciones discrecionales sobre las ayudas automáticas (basadas en indicadores estadísticos como el valor de la recaudación, el tiraje de la publicación u otras fórmulas más o menos complejas). Los sectores más estructurados (como la industria del cine o el libro) o los actores más institucionales (municipios o equipamientos que forman parte de redes) han conseguido imponer sistemas automáticos, más transparentes y predecibles (aunque a menudo beneficien a los agentes más poderosos). De igual forma, el predominio de la subvención anual se explica por la escasa institucionalización de las políticas, aunque en la última década ha aumentado el número de programas-contrato con sistemas de subvención plurianuales. En este aspecto, Francia lleva bastantes años de ventaja a su vecino meridional.

Pero es en la subvención directa a pequeños proyectos culturales y a los creadores individuales donde se vislumbra mejor la tensión entre el viejo clientelismo político y la delegación en profesionales especializados. No son cuestiones fáciles, pues más allá de las opciones estéticas, la identidad y el valor simbólico juegan un indudable peso en la decisión política. La mayor consolidación de la estructura administrativa, de la cultura democrática y del sistema de profesionales culturales franceses explican el menor peso del clientelismo político al norte de los Pirineos. El papel de la administración central como subvencionadora de otras estructuras públicas o de apoyo a la iniciativa privada, es cada vez menos importante no solo en España sino también en Francia. Esta es otra convergencia entre modelos, en la que la territorialización cada vez es más determinante en el desarrollo de las políticas culturales. De todas formas, tal como se analiza más adelante, las lógicas de distribución y las potenciales relaciones clientelares son claramente distintas entre el nivel local y el nivel estatal.

Las estrategias de información y mediación toman una importancia creciente en las políticas culturales contemporáneas, pero están mucho más evolucionadas en Francia que en España. Más allá de la tradicional usanza de los reconocimientos públicos (premios u honores) o del creciente uso de Internet y de estrategias de ventanilla única para dar a conocer y facilitar la tarea de los actores culturales, el Ministerio de Cultura francés ha potenciado los sellos o marcas de calidad institucional. De forma parecida a una franquicia comercial, el Ministerio define las características y modelos de gestión que deben seguir cada uno de los equipamientos que quieran merecer un determinado sello de calidad y beneficiarse de la subvención condicionada que lo acompaña. Así, las diversas ciudades o regiones compiten entre ellas para conseguir 
un centro dramático o coreográfico nacional, un Zénith para acoger los grandes conciertos populares, una escena de música actual o cualquier otra de las marcas dispo-

nibles. Este instrumento, de carácter mucho más indirecto pero no menos eficiente, solo se explica por la supremacía institucional y el reconocimiento conceptual y simbólico del Ministerio sobre los restantes niveles de gobierno. Las administraciones territoriales luchan por el prestigio y los recursos financieros del Estado, y éste impone de forma subliminal su particular concepción de los modelos de intervención cultural. Esta imposición se completa con la alianza con los responsables de los distintos equipamientos, ya que poder circular por la red francesa de centros equivalentes les permite progresar en la carrera profesional (Dubois, 2010).

Este instrumento no es posible en España dada la alta autonomía política y administrativa de las administraciones territoriales (y de las propias instituciones culturales), la inexistencia de un sistema profesional cohesionado (más allá de los cuerpos de bibliotecarios o archiveros) y la incapacidad del Ministerio para desarrollar este tipo de programas. Desde su estructuración en la época de Javier Solana (1982-88), el Ministerio se ha centrado en los equipamientos o elencos nacionales, para el realce de la capital del Estado, o en apoyar determinados intereses sectoriales (Bouzada, 2007).

Finalmente, la dimensión de inspección y sanción en las políticas culturales tiene un largo recorrido histórico a través de la imposición y la censura. Sin embargo, más allá de la protección del patrimonio, es escasamente utilizada por su potencial carácter represor sobre la libertad de expresión, hecho especialmente sensible tanto en un país de larga experiencia dictatorial como en la cuna de los derechos humanos.

\section{C) Distribución de responsabilidades y competencias entre niveles de gobierno}

El análisis de los presupuestos públicos por niveles de gobierno, programas y sectores, permite distinguir las grandes prioridades de las políticas de cultura contrastándolas con las competencias formales de cada nivel. Desgraciadamente, es extraordinariamente difícil disponer de datos presupuestarios comparables a escala internacional, pues los criterios contables y de asignación de los mismos son muy heterogéneos. Sin embargo, una estimación aproximada del gasto cultural directo aporta datos suficientemente ilustrativos sobre las diferencias de modelo territorial entre España y Francia.

El gasto cultural francés duplica en valores absolutos al español, aunque el primero incluye competencias sobre comunicación. Mientras que en España la aportación de la administración central representa el 14\% del total (la mayor parte de ellos administrados por el Ministerio de Cultura), en Francia alcanza el 51\% del gasto no consolidado, cuyo $60 \%$ es aportado por otros departamentos del gobierno (educación, primer ministro o asuntos extranjeros). En contrapartida, mientras que en Espa64 ña las Comunidades Autónomas contribuyen con el $27 \%$ del presupuesto total, en 
Francia los consejos regionales únicamente representan el 4\%. Cabe tener en cuenta que el Ministerio de Cultura francés mantiene potentes direcciones regionales desde las que despliega su política de cooperación con las administraciones territoriales y los actores culturales locales.

TABLA 1

Estimación del presupuesto en cultura de España y Francia, por niveles de gobierno*

\begin{tabular}{|l|c|c|c|c|}
\hline \multirow{2}{*}{} & \multicolumn{2}{|c|}{ España } & \multicolumn{2}{c|}{ Francia } \\
\cline { 2 - 5 } & Millones $€$ & $(\%)$ & Millones $€$ & $(\%)$ \\
\hline Administración central & 1.075 & 14 & 7.453 & 51 \\
\hline Administraciones regionales & 2.129 & 27 & 556 & 4 \\
\hline Administraciones provinciales & 669 & 9 & 842 & 6 \\
\hline Administraciones locales & 3.907 & 50 & 5.649 & 39 \\
\hline Total no consolidado & 7.779 & 100 & 4.500 & 100 \\
\hline
\end{tabular}

* Los datos españoles corresponden a la liquidación del presupuesto del año 2008, mientras que en el caso francés los datos son más heterogéneos (ver notas metodológicas en Ministère de la Culture et de la Communication, 2010).

Fuente: Ministerio de Cultura (2010) y Ministère de la Culture et de la Communication (2010).

Un análisis pormenorizado de las políticas regionales españolas y francesas daría como resultado una enorme heterogeneidad de estrategias de intervención. No se trata únicamente del gran diferencial de recursos disponibles o del grado de autonomía política formal existente, sino también de la conciencia identitaria de cada una de ellas. Las políticas culturales releen el patrimonio, construyen identidad y abren a la modernidad en función de parámetros ideológicos y morfológicos. Regiones con idioma y conciencia nacional propia tienden a desplegar políticas culturales mucho más autónomas que regiones constituidas de forma más o menos artificial desde las oficinas de planificación nacional. Tampoco es lo mismo en función del partido que gobierne y el carácter más o menos urbano o rural de cada región.

Finalmente, los poderes locales se han convertido en unos actores claves de las políticas culturales durante el último cuarto de siglo en ambos países. En el caso español, los municipios han ejercido su autonomía de gasto para centrar buena parte de sus recursos en servicios personales. En Francia, a esta tendencia general se le suma la estrategia de la administración central de firmar acuerdos de cooperación con los otros niveles de administración. Los municipios (junto a las comunidades de aglomeración en el caso francés) asumen la mayoría de responsabilidades en ámbitos fundamentales de la acción cultural: lectura pública, artes escénicas, patrimonio, archivos, educación artística extra-escolar y un largo etcétera. Para ello se han dotado de nuevas generaciones de equipamientos culturales y multitud de programas. De todas formas, mientras que en Francia la situación financiera de los municipios está más saneada que los consejos de región y departamento, en Espa- 
ña es con la crisis el nivel de gobierno con mayores dificultades para dar respuesta a las expectativas ciudadanas generadas.

\section{LAS ESTRATEGIAS DE LEGITIMACIÓN Y EFICIENCIA EN LOS PROCESOS DE ESTANDARIZACIÓN O DIFERENCIACIÓN DE LAS POLIITICAS CULTURALES}

La dialéctica entre diferenciación y estandarización es el resultado de la tensión entre objetivos de legitimación y eficiencia en el ámbito de las políticas públicas, y de la capacidad de incidencia sobre los mismos de distintas categorías de actores. En momentos de gran transformación de los modelos, como durante la transición política española o en el momento actual de grave crisis económica, la lucha para imponer un nuevo marco es más abierta, al igual que la aparición de nuevos argumentos que la legitimen. En épocas más tranquilas, la dialéctica subyacente a los procesos de estandarización o diferenciación son más sutiles, con mayor peso de los argumentos de eficiencia.

Cuatro grandes lógicas interactúan entre sí para imponer un discurso políticocultural favorable a sus intereses. En primer lugar, el sistema institucional y sus agentes. Éste tiende a homogeneizar los valores y las normas, pues potencia el status quo de la administración como sistema, y de los altos funcionarios en particular. La segunda gran lógica responde a las fuerzas del mercado, formada por empresas e intereses gremiales de tamaño y capacidad de incidencia heterogéneas en la construcción de discurso. La tercera lógica corresponde al sector no lucrativo, formado por el tejido de asociaciones, valores y prácticas de la sociedad civil. Finalmente, la cuarta lógica se construye alrededor de la política, las ideologías y los partidos políticos.

\section{GRÁFICO 1}

La construcción dialéctica del discurso político-cultural, y su efecto estandarizacióndiferenciación, por parte de las cuatro grandes lógicas en juego

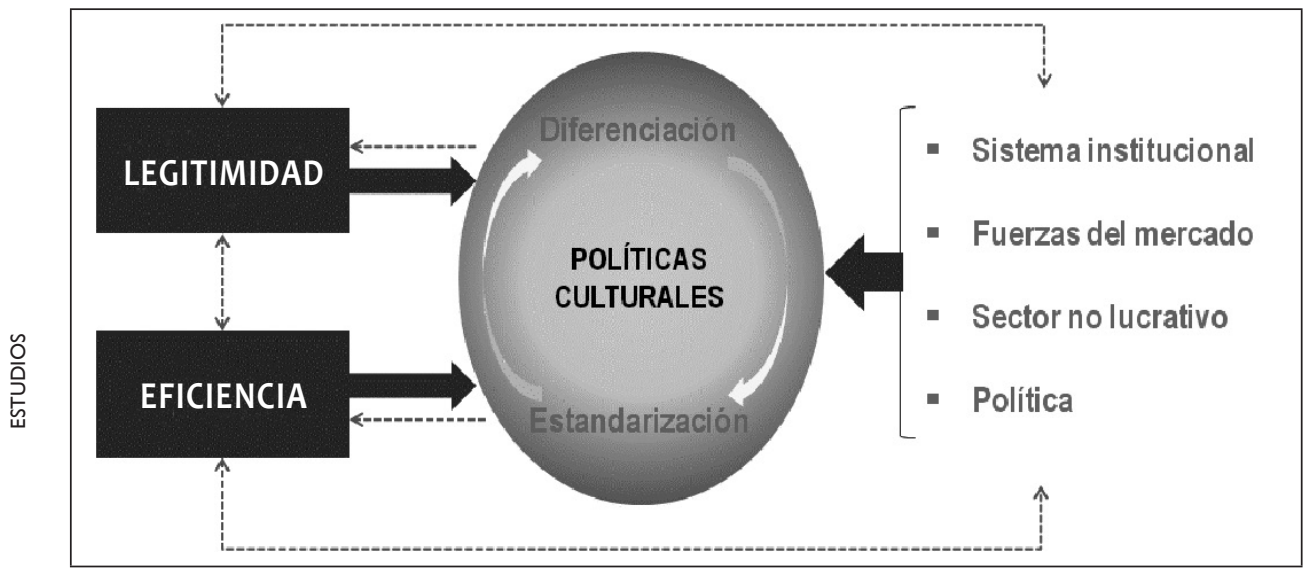

Fuente: Bonet, L. y Négrier, E. (2010). 
En el caso español, existe una tensión entre la lógica del sistema institucional, que tiende más hacia la diferenciación como consecuencia del alto grado de autonomía política y administrativa de los entes territoriales, y la lógica administrativa que presiona hacia la estandarización. De todas formas, factores que podrían responder a lógicas de diferenciación en la práctica terminan por ser vectores de homogeneización, y viceversa. Así pues, gobiernos con responsabilidades semejantes tienden a la importación o transferencia de instrumentos o "buenas prácticas" (Radaelli y Schmidt, 2004), el isomorfismo mimético de Powell y DiMaggio. Se genera una interdependencia entre el elemento de transferencia (el modelo de acción) y los elementos no negociables (cultura política, nivel de desarrollo económico o ubicación geográfica) que, paradójicamente, hace posible la transferencia. Por ejemplo, muchas instituciones y políticos locales y regionales interesados en sobresalir tienden a copiar fórmulas de éxito de otros gobiernos, con el resultado de una mayor homologación entre las prácticas. Pero esta tendencia no es sistemática. En el caso francés, la aplicación coercitiva de las nuevas normas centralizadoras puede producir mayores diferencias regionales, a pesar de que esta regulación pretendía la homologación y se inspiraron en los valores de igualdad territorial (Négrier, 2003).

Por su lado, los agentes culturales privados y las fuerzas de mercado acostumbran a concentrar sus esfuerzos en la eliminación de los obstáculos políticos y regulatorios para la libre circulación de los factores productivos y las mercancías. Sin embargo, los intereses reales de cada grupo de poder pueden contradecir dicha regla. El perfil de las clientelas y, por lo tanto, de los intereses a defender cambia radicalmente si se trata de un gobierno local o regional (que recibe presiones para proteger el tejido empresarial y profesional local) o si se trata del gobierno central (presionado para abrir la competencia por parte de las grandes corporaciones). La connivencia entre gobierno central y grandes grupos de cultura y comunicación es patente cada vez que se modifica una regulación del mercado, los criterios de subvención o los beneficios fiscales (Bonet y Négrier, 2010); y de forma parecida, en los niveles de gobierno inferior con las empresas de su órbita. El sector del libro o del cine en España permiten ilustrar detalladamente la complicidad entre poderes públicos y sector empresarial, desde la designación de ministros o directores generales "de la casa", hasta la formulación de propuestas legislativas en los despachos de las asociaciones profesionales. Otra cosa es la retórica pública, que justifica los intereses particulares como parte del interés general. El mercado, que en general tiende a la estandarización, se transforma así en un vector de diferenciación más de las políticas públicas.

La tercera categoría está formada por el sector no lucrativo. Mucho mayor en tamaño que el mundo empresarial, su gran fragmentación le dificulta incidir en el diseño de las políticas públicas. Solo ocasionalmente consigue unirse para defender posturas comunes, como en el reciente Pacto por la cultura ante la crisis y el recorte presupuestario'. En Francia, una tradición asociativa mucho más potente consigue

Ver <http://www.conferenciadelacultura.net> (consulta 15 enero 2011). 
que los encuentros asociativos sean más frecuentes e influyentes. De todas formas, la heterogeneidad de realidades sectoriales y territoriales, genera que su influencia se circunscriba fundamentalmente al nivel local.

La última lógica en juego la constituyen las ideologías y sus principales actores: los partidos políticos. Cabría esperar una tendencia clara hacia la diferenciación de propuestas, pero de nuevo la realidad es más compleja dado el doble eje de referencia ideológico: derecha-izquierda, e identidad nacional central frente a periférica. Por un lado, la retórica de los partidos conservadores es favorable a la reducción de la presencia del Estado, con políticas más orientadas al fomento de la demanda que de la oferta cultural. En la práctica, los presupuestos y las directrices se mantienen con escasos cambios, quizás con menos apoyo a las artes emergentes o a las estrategias sociales. El caso español muestra cómo el tránsito de gabinetes socialistas a populares no implicaron transformaciones significativas en las políticas culturales estatales (Rubio Aróstegui, 2003, 2005 y 2008). Algo parecido se da en Francia, donde la estrategia de algunas personalidades desplaza ampliamente a las ideologías (Dubois, 2010). Solo cuando la extrema derecha toma el poder se da un cambio radical que genera, como ya se ha comentado, un grave desencuentro entre la esfera política y la técnica (Bressat-Bodet, 1999).

El segundo eje condiciona ideológicamente mucho más la praxis de la política cultural, pues la cuestión de la identidad territorial es hoy tan sensible en la federalizante España como en la centralista Francia. La incidencia de componentes nacionalistas de carácter centrípeto (español y francés) en la configuración ideológica de los grandes partidos estatales dificulta la reducción del papel del Estado por parte de las fuerzas conservadoras. La alternancia derecha-izquierda casi no cambia las políticas nacionales de prestigio o la acción cultural exterior. En cambio, en el caso español, las transformaciones más radicales se dan cuando cambia la sensibilidad nacional de los gobiernos autonómicos, tal como ha pasado en la Comunidad Valenciana, el País Vasco o las Islas Baleares. En Francia, existe un amplio consenso ideológico "republicano" sobre el concepto de identidad nacional y su influencia en las políticas culturales. Es patente en la acción de los diversos niveles de gobierno y se refuerza desde la red de direcciones regionales de asuntos culturales del Ministerio de Cultura, con recursos y capacidad de influencia. Pero mientras la identidad regional tenía tradicionalmente un sentido peyorativo, hoy la capacidad de diferenciación regional es expresión de modernidad. De todas formas, las diferencias en las prioridades regionales responden tanto a razones de dinamismo socioeconómico y demográfico -rural, urbano, grandes metrópolis- como a la reivindicación de las tradiciones regionales, tal como se observa en regiones como Alsacia (Négrier y Teillet, 2011).

En términos generales, el paisaje de las políticas culturales franco-españolas muestra la simultaneidad de tendencias homogeneizadoras y diferenciadoras, con independencia de su origen o lógica tractora. Su legitimación se asienta en argumentos diversos, construidos socialmente a lo largo de décadas. El proceso de globaliza68 ción, así como la trasformación de los formatos de producción, mediación y consumo 
cultural propulsado por la revolución digital, representa un reto para muchos de los paradigmas tradicionales, pero su respuesta es a la vez global y local.

Evidentemente, buena parte de la propensión hacia la homologación tiene su origen en la capacidad normativa y de construcción conceptual de los organismos nacionales y supranacionales, legitimados en buena medida por el proceso de globalización. Este sería el caso de la regulación sobre la competencia, o los esfuerzos para racionalizar las políticas gubernamentales de la nueva gestión pública (Bezes, 2009), con derivaciones descendientes desde el nivel europeo al local. Su efecto sobre el sector cultural es más que notable, pues al mismo tiempo que incrementan la transparencia y la eficiencia, condiciona las medidas de apoyo y protección a las culturas locales. En el plano de los argumentos de legitimación, también se avanza hacia una mayor homologación en la medida que las instituciones europeas fortalecen el discurso de otros gobiernos en favor de políticas públicas de apoyo a la economía creativo-cultural, más allá de su justificación intrínseca. Otro aspecto no menor es la homologación de referencias técnicas o profesionales, pues al lado de la regulación el paradigma se expande a través de las redes de intereses o influencias profesionales o políticas.

Pero los factores de diferenciación no se quedan cortos. Por un lado, la heterogeneidad estructural del territorio conduce a una pluralidad de estrategias. Las pequeñas regiones rurales necesitan políticas adaptadas a su realidad, muy distintas de las implementadas en las grandes metrópolis. Por esto el principio de subsidiaridad se adapta tan bien a las políticas culturales. Además, ciudades demográfica o socialmente parecidas pueden tomar opciones claramente distintas, pues tan válidas son la danza como las artes plásticas para avanzar en el desarrollo cultural. Su mayor o menor empuje es discrecional, fruto de tradiciones u oportunidades coyunturales que con el tiempo devienen estructurales. A diferencia de otras políticas sociales, las políticas culturales se caracterizan por su componente voluntarista, relacionado con la intensidad de la generación y la apropiación simbólica de las distintas propuestas. La profesionalización de las políticas culturales, que por un lado estandariza procesos, termina por diseñar estrategias a la medida de cada lugar y momento.

Los valores, los paradigmas y los objetivos de política cultural están en plena transformación. Las relaciones tradicionales de fuerza y las alianzas entre los actores culturales y no culturales con intereses en el sector -desde las empresas de telefonía a las gestoras de derecho de autor, pasando por las distintas asociaciones de empresas y profesionales de la cultura- cambian continuamente. Nuevas estrategias se mezclan con viejos instrumentos dando como resultado unas políticas globalmente incoherentes. En este contexto se acentúan los intentos de copiar buenas prácticas, sin que nadie garantice el resultado deseado. El diseño de políticas cada vez más orientadas a la consecución de objetivos y a la eficiencia desdibuja las viejas retóricas legitimadoras. Solamente la evaluación continua de los resultados obtenidos con los recursos disponibles permite aproximar la praxis de los programas con las grandes finalidades políticas. 


\section{CONCLUSIONES}

La excesiva simplificación de la clasificación propuesta por Chartrand y McCaughey no permite profundizar en el complejo sistema que constituye una política cultural contemporánea, de la construcción de paradigmas a la definición de estrategias e implementación de instrumentos. En un mundo globalizado, las tendencias homogeneizadoras son crecientes, pero la tensión entre diferenciación y estandarización persiste, tanto entre la realidad nacional y la supranacional, como entre la primera y los niveles subestatales.

El análisis comparado de las políticas culturales franco-españolas demuestra que no es posible aceptar la hipótesis de la singularidad y coherencia nacional. Las políticas públicas no se construyen solo como resultado del juego combinado de paradigmas, formas de organización y sistemas profesionales construidos históricamente en un particular contexto nacional. Pero tampoco es cierta la hipótesis contraria, aquella que explicaría que los sistemas nacionales son un subsistema del modelo europeocomunitario. Esta es una de las razones que explican que en el seno de las políticas armonizadas a escala de la Unión se mantenga un gran número de particularidades nacionales, tal como denotan las transposiciones de las directivas comunitarias a la legislación de cada estado. Los factores nacionales que homogeneízan las distintas políticas públicas y sus modelos de gestión son evidentes, pero, como se ha comentado, se relativizan por fenómenos de diferenciación interna hacia abajo y por la hibridación externa a escala internacional.

A lo largo del artículo hemos mostrado diversas evidencias que permiten constatar que muchas lógicas o factores que a priori son homogeneizantes pueden favorecer la diferenciación, y viceversa. La dialéctica entre diferenciación y estandarización, como marca de modernidad de las políticas culturales, permite explicar el fuerte desarrollo a escala territorial de las estrategias de benchmarking. Éstas muestran, paradójicamente, que "aquello que te distingue de los demás es lo que te permite ser comparado". Asimismo, los aspectos de diferenciación interna son los que terminan por ser más convergentes a escala internacional, mientras que aquellos que los empujan hacia la estandarización interna generan más diferencias en la comparación internacional. Esto nos llevaría a aceptar la fortaleza de los marcos nacionales o subestales históricos aun y el acelerado e intenso proceso de interacción a escala internacional.

Así pues, la doble tendencia hacia la diferenciación o hacia la estandarización no se limita al diálogo/tensión entre los Estados y los organismos supranacionales, sino que integran asimismo al conjunto de interacciones con los niveles sub-estatales. La comparación entre Francia y España, dos países que comparten una misma tradición institucional pero con estructuras de distribución de competencias entre niveles de gobierno marcadamente distintas, demuestra que en cada nivel de gobierno se dan 70 tendencias en ambas direcciones. 


\section{REFERENCIAS BIBLIOGRÁFICAS Y DOCUMENTALES}

Ariño, A., Bouzada, X. y Rodríguez, A. (2005), “Políticas culturales en España”, en J.A. Roche y M. Oliver, eds., Cultura y globalización. Entre el conflicto y el diálogo. Alicante: Publicaciones de la Universidad de Alicante.

Audet, C. y Saint-Pierre, D., eds. (2010), Tendances et Défis des Politiques Culturelles. Cas nationaux en perspective. Quebec: Presses de l'Université Laval.

Belfiore, E. (2004), "The methodological challenge of cross-national research: comparing cultural policy in Britain and Italy", CCPS Online Publications, Coventry: Centre for Cultural Policy Studies. En línea: <http://www2.warwick.ac.uk/fac/ arts/theatre_s/cp/publications/centrepubs/ccps_paper_8.pdf> (consulta: $20 \mathrm{di}$ ciembre de 2010).

Benhamou, F. (2006), Les dérèglements de l'exception culturelle. París: Éditions du Seuil.

Bezes, P. (2009), Réinventer l'État. Les réformes de l'administration française (1962 2008). París: PUF.

Bonet, L. y Négrier, E. (2011), “Un modèle espagnol de politique culturelle?”, en P. Poirrier, ed., Histoire comparée des politiques culturelles. París: La Documentation Française.

Bonet, L. y Négrier, E. (2010), “Cultural policy in Spain: Processes and Dialectics”, Cultural Trends 19(1-2): 41-51.

Bonet, L. y Négrier, E., eds. (2008), La fin des culturelles nationales? Les politiques culturelles à l'épreuve de la diversité. París: La Découverte.

Bouzada, X. (2007), “La gouvernance de la culture en Espagne”, en L. Bonet y E. Négrier, eds., La politique culturelle en Espagne. París: Khartala.

Bressat-Bodet, C. (1999), "Culture et autorité partisane: la politique de «rééquilibrage» de la bibliothèque d'une municipalité FN (Orange, 1995-1997)", Pôle Sud, 10: 75-92.

Chartrand, H. y McCaughey, C. (1989), “The Arm's Length Principle and the Arts: An International Perspective - Past, Present and Future", en M.C. Cummings y J.M. Schuster, eds., Who's to Pay for the Arts? The International Search for Models of Support. Nueva York: ACA Books.

Cummings, M.C. y Katz, R.S., eds. (1987), The Patron State: Government and the Arts in Industrialized Democracies. Nueva York: Oxford University Press.

Delvainquière, J-C. (2010), "France”, Compendium of Cultural Policies and Trends in Europe. En línea <http://www.culturalpolicies.net/web/france.php> (consulta: 20 diciembre 2010).

Dezalay, Y. y Garth, B. (2002), “Legitimating the New Legal Orthodoxy”, en Y. Dezalay y B. Garth, eds., Global Prescription. The Production, Exportation, and Importation of a New Legal Orthodoxy. Ann Arbor: University of Michigan Press.

DiMaggio P.J. y Powell W. (1991), “The Iron Cage Revisited: Institutional Isomorphism and Collective Rationality in Organizational Fields", en W. Powell y P. DiMaggio, eds., The New Institutionalism in Organizational Analysis. Chicago: University of Chicago Press.

Drezner, D. (2005), “Globalization, Harmonization, and Competition: The Different Pathways to Policy Convergence”, Journal of European Public Policy, 12(5): 841-859. 
Dubois, V. (2010), "Le modèle français et sa crise: ambitions, ambiguïtés et défis d'une politique culturelle", en C. Audet y D. Saint-Pierre, eds., Tendances et Défis des Politiques Culturelles. Cas nationaux en perspective. Quebec: Presses de I'Université Laval.

Dunn, W. y Miller, D. (2007), "A Critique of the New Public Management and the NeoWeberian State: Advancing a Critical Theory of Administrative Reform", Public Organization Review, 7 (4): 345-358.

Dubois, V. y Négrier, E. (1999), "L'institutionnalisation des politiques culturelles en Europe du Sud: éléments pour une approche comparée", Pôle Sud, 10: 5-9.

Evans, M., ed. (2004), Policy Transfer in Global Perspective. Aldershot: Ashgate.

Fligstein, N. (2008), Euro-Clash. The EU, European Identity and the Future of Europe, Oxford: Oxford University Press.

Gomà, R. y Subirats, J. (1998), Políticas públicas en España: contenidos, redes de actores y niveles de gobierno. Barcelona: Ariel.

Gray, C. (1996), “Comparing cultural policy: a reformulation”, European Journal of Cultural Policy, 2(2): 213-222.

Hood, C. (2000), The Art of the State: Culture, Rhetoric and Public Management. Oxford: Clarendon.

Kassim H., Menon, A., Peters, G. y Wright, V., eds. (2001), The national co-ordination of EU policy: the European level; the domestic level, 2 vol. Nueva York/Oxford: Oxford University Press.

Knill, C. (1998), "European Policies: The Impact of National Administrative Traditions", Journal of Public Policy, 18(1): 1-28.

Knill, C. y Lahmkuhl, D. (2002), "The national impact of European Union regulatory policy: Three Europeanization mechanisms”, European Journal of Political Research, 41: 255-280.

Lenschow, A. Liefferink, D. y Veenman, S. (2005), "When the Birds Sing: A Framework for Analysing Domestic Factors behind Policy Convergence", Journal of European Public Policy, 12(5): 797-816.

Ministère de la Culture et de la Communication (2010) "Chiffres clées 2010. Statistiques de la culture”. En línea <http://www2.culture.gouv.fr/culture/deps/chiffrescles2010/20-financement-2010.pdf> (consulta: 15 enero 2011).

Ministerio de Cultura (2010), "Estadística de Financiación y Gasto Público en Cultura”. En línea <http://www.mcu.es/culturabase/cgi/um? M=/t3/p3\&O=culturabase \&N= $\& L=0>$ (consulta: 11 enero 2011).

Moreno, L. (2001), The Federalization of Spain. Londres/Portland: Frank Cass/Routledge. Muller, P. (2009), Les politiques publiques, París: PUF.

Négrier, E. (2003), "Politiques culturelles territoriales: dernier inventaire avant décentralisation?”, L'Annuaire des collectivités locales GRALE. París: CNRS Editions.

Négrier, E. y Teillet, P. (2011), “La question régionale en Culture”, en S. Barone, dir., Les politiques régionales en France. París: La Découverte.

Prieto de Pedro, J. (1995), Cultura, Culturas y Constitución. Madrid: Editorial Centro de Estudios Culturales.

Radaelli, C. (2003), "The Europeanization of Public Policy”, en K. Featherstone y C. 72 Radaelli, eds., The Politics of Europeanization. Oxford: Oxford University Press. 
Radaelli, C. y Schmidt, V. (2004), "Policy Change and Discourses in Europe: Conceptual and Methodological Issues", West European Politics, 27(2): 183-210.

Rubio Aróstegui, J.A. (2008), “Niveles de discurso de la política cultural y sus interacciones en la construcción de la realidad artística y cultural”, Periférica, 9: 21-40.

Rubio Aróstegui, J.A. (2005), “Las contradicciones de la política cultural del Estado en los gobiernos populares: entre el ¿liberalismo? y el continuismo socialista”, Sistema, 187: 111-124.

Rubio Aróstegui, J.A. (2003), La política cultural del Estado en los gobiernos socialistas: 1982-1996. Gijón: Trea.

Saurruger, S. y Surel, Y. (2006), “L'européanisation comme processus de transfert de politique publique", Revue internationale de politique comparée, 13(2): 179-211.

Sen, A. (1989), "Development as Capabilities Expansion”, Journal of Development Planning, 19: 41-58.

Vestheim, G. (2009), "The Autonomy of Culture and the Arts: From the Early Bourgeois Era to Late Modern 'Runaway World”', en M. Pyykkönen, N. Simanainen y S. Soka, eds., What about Cultural Policy? Jyväskylä: Minerva.

Villarroya, A. (2010), "Spain”, Compendium of Cultural Policies and Trends in Europe. En línea <http://www.culturalpolicies.net/web/spain.php> (consulta: 20 diciembre 2010).

Wallace, H. y Wallace, W., eds. (2000), Policy-making in the European Union. Oxford: Oxford University Press.

Recibido: 31 de enero de 2011

Aceptado: 1 de junio de 2011 
